\title{
Pain measurement techniques: spotlight on mechanically ventilated patients
}

This article was published in the following Dove Press journal: Journal of Pain Research

\author{
Isabela Freire Azevedo- \\ Santos \\ Josimari Melo DeSantana \\ Neuroscience Research Laboratory \\ (LAPENE), Department of Physical \\ Therapy, Graduate Program of \\ Health Science, Graduate Program \\ of Physiological Science, Federal \\ University of Sergipe, Aracaju, Sergipe, \\ Brazil
}

Background: Procedural pain is a frequent problem in intensive care units (ICUs). For that, pain assessment has been increasingly introduced to the ICU professional's routine, and studies have been developed to show the relevance of measuring pain in critically ill patients.

Objective: This review aimed to describe pain measurement techniques for mechanically ventilated adult patients based on evidence and already published.

Method: Systematic literature search was performed on PubMed and Google Scholar. Keywords "pain", "pain measurement", "intensive care units" and "respiration, artificial" were combined to the Boolean operator AND. No language or publication year was limited in this search. The purpose and method of all papers were analyzed and only studies which described pain assessment in mechanically ventilated patients were included in this review.

Results: Objective methods were found in the literature to assess pain in mechanically ventilated adults. Behavioral scales were the most used method for pain measurement in noncommunicative patients. Vital signs were used, but the reliability of this method was questioned. Pupillometry, bispectral index and skin conductance were found and described as pain assessment methods. Conclusion: This review showed that objective measures, as behavioral scales, are the gold standard tools to measure pain intensity in noncommunicative subjects. These data contribute to professionals' knowledge about ICU pain measurement and emphasize its importance and consequences for adequate pain management.

Keywords: pain, pain measurement, intensive care units, respiration, artificial

\section{Introduction}

Intensive care, or critical care, is a health specialty dedicated to multidisciplinary management of patients with acute organ dysfunction. ${ }^{1}$ The main objective of intensive care is to prevent further physiologic deterioration by the treatment and solution of acute and/or severe diseases and to save life during a life-threatening condition. ${ }^{1}$ For that, intensive care unit (ICU) is the environment organized to meet the needs of these critically ill and mechanically ventilated patients, once their care involves a specific physical space, with support, monitoring technology, and specialized human resources. ${ }^{2}$

In ICUs, three important concepts are commonly used: pain, agitation and delirium. ${ }^{3}$ Pain is "an unpleasant sensory and emotional experience associated with actual or potential tissue damage, or described in terms of such damage". ${ }^{3,4}$ Agitation and anxiety commonly occur in ICUs and are associated with adverse clinical outcomes, such as hypoxemia, hypotension, and/or withdrawal from alcohol and other substances. ${ }^{3}$ Lastly, delirium is known as "an acute onset of brain dysfunction, characterized by level of 
consciousness disturbance and cognition changes (memory deficit, disorientation, language disturbance)". ${ }^{3}$ Despite pain, delirium and agitation are interconnected, pain is the most neglected sign in ICUs.

Analgesics and sedatives are medicines commonly used in ICUs with the objective to maintain comfort, relieve anxiety, facilitate care and adapt patients to ventilatory support. ${ }^{5}$ Some sedation protocols emphasize lighter use of sedatives for mechanically ventilated patients, daily sedation interruption and analgesia based on sedation protocols, which means that analgesic administration occurs and then adding sedation if required. ${ }^{6-12}$ Currently, clinicians observed that the primary goal in ICUs should be pain and discomfort control, and then, if necessary, sedation should be performed. To achieve this goal, analgo-sedation protocols have been developed and applied in ICU patients. ${ }^{12}$ Analgo-sedation protocols that have been introduced into practice decrease duration of invasive mechanical ventilation (IMV), ventilator-associated pneumonia incidence and improve the probability of successful extubation. ${ }^{13-15}$

Despite the fact that pain in critically ill and mechanically ventilated patients has been studied over the last 20 years, this sign is present in about $50 \%$ of this population. ${ }^{3}$ One of the main causes of pain in the ICU is the medical procedures, unavoidable and necessary actions that are responsible for changes in pain intensity compared to rest. ${ }^{16}$ A study performed in Europe (The Europain ${ }^{\circledR}$ Study) observed the increase on pain intensity during 12 procedures, such as chest tube removal, peripheral intravenous insertion, wound care, mobilization, positioning, respiratory exercises and others. ${ }^{16}$ Among these routine activities, tracheal suctioning was considered the most painful, responsible for certain behaviors (grimace, facial responses and clenched fists) and changes on physiological parameters. ${ }^{16,17}$

Inadequate procedural pain treatment is a problem in the ICU, and inadequate treatment of this sign remains as a lack in the clinical setting. ${ }^{3}$ Barriers on pain management are associated to difficulties on assessment, since pain has a subjective nature and it is understood as a variable that can be measured only when reported by the person experiencing it. ${ }^{3}$ The majority of patients undergoing intensive care are unable to self-report their pain because of low consciousness levels, sedatives or neuromuscular blocking agents use and IMV..$^{3,18}$

From the observation of these subjects unable to selfreport, the International Association for the Study of Pain (IASP) registered that "the inability to communicate verbally does not negate the possibility that an individual is experiencing pain and is in need of appropriate pain-relieving treatment". ${ }^{4}$ For that, pain assessment has been increasingly introduced to the ICU professional's routine and studies have been developed to show the relevance of measuring this sign in critically ill patients.

A large number of instruments can be used for pain assessment of unconscious and mechanically ventilated patients. It is based on behaviors, observation, physiological parameters, and other body signs that can indicate nociception. ${ }^{19}$ From that, this review aimed to describe pain measurement techniques for mechanically ventilated adult patients based on evidences and perspectives already published about this subject.

This narrative review was based on a systematic literature search performed on PubMed and Google Scholar. The same results were obtained in both databases. Keywords "pain", "pain measurement", "intensive care units" and "respiration, artificial" were combined to the Boolean operator AND. No language or publication year was limited on this search and all available papers were analyzed. If full text was unavailable, corresponding authors were contacted by email. The purpose and method of all papers were analyzed and only studies which described pain assessment in critically ill patients were included in this review.

\section{Pain assessment in ICUs}

In the last two decades, the American Pain Society has established "Pain as the fifth vital sign" initiative. This proposal consists of professional's awareness about the introduction of pain assessment in addition to other vital signs measurement, such as blood pressure, pulse, temperature, and respiration. ${ }^{20}$ This pain assessment task is indicated for all health professionals that directly care for critically ill patients. Nurses, clinicians, physical therapists, and other caregivers must use structured, valid, reliable, and feasible tools to assess pain in the ICU, in a routine and repetitive manner. ${ }^{3}$

The importance of this routine on critically ill patient's outcomes is proven by studies that report its impact in ICUs. It is evidenced that the establishment of pain assessment protocols is responsible for better pain management, more efficient use of analgesics and/or sedatives, decrease in IMV duration, increased odds for weaning from IMV, lower risk of ventilator-associated pneumonia, central catheter-related infections, urinary tract infections, and bacteremia development, shorter duration of ICU stay and decrease in agitation events and mortality rate. ${ }^{21-23}$ Based on those positive outcomes, pain assessment is considered a strategy for a better ICU care. Behavior analysis and vital sign records are the most used methods for this practice. These and other pain measurement instruments will be described below. 


\section{Behavioral scales}

Mechanically ventilated patients who are unconscious and unable to self-report, require instruments that detects behavior details associated to pain perception and expression. Facial expressions observation is the main method for pain assessment in ventilated subjects. ${ }^{24}$ It is known that during painful procedures, there is an increase in facial movements - eyes closed, brow lowered, lid tightened, cheeks raised and lips parted. ${ }^{25}$ In addition to that, body movements, muscle rigidity, compliance with mechanical ventilator and/or sounds are other behavior indicators of pain intensity in ICU patients. ${ }^{24}$

Based on these pain indicators, behavior assessment scales were developed and validated to mechanically ventilated patients. These instruments can be either unidimensional or multidimensional, depending on the topics considered. Unidimensional scales are composed of a single dimension (behavior responses, for example) and can consider one (facial expressions, for example) or multiple domains (facial expressions, body movements and sounds). ${ }^{26}$ Alternatively, multidimensional scales evaluate two or more pain dimensions (behaviors, physiological responses) and have several domains within each dimension. ${ }^{26}$

From nine scales validated for critically ill adults, six are unidimensional and three multidimensional. ${ }^{26-28}$ Behavior assessment is the characteristic considered in most of these instruments, once it is the recommended method for pain measurement in subjects unable to self-report. ${ }^{24}$ The other multidimensional scales are composed of behavior and vital signs data, also based on clinical judgement of nurses or other health professionals. ${ }^{27}$

Previous scores were determined for each of these scales, with minimum, maximum and cut-off values that indicate the presence or absence of pain. It is important to notice that behavior pain scores based on patient observation should be interpreted differently from self-report. Although both of these measures represent pain scores, observation is associated with behavioral responses exhibited by patients to express their pain, while self-report relates to the sensory dimension of pain which refers to the perception of this sign by the person who is experiencing it. ${ }^{24} \mathrm{It}$ is known that pain intensity scores and behavioral responses are directly proportional, but they are not equivalent. ${ }^{27}$ For that, only validated and specific instruments must be used for pain assessment in ICUs.

Scale development and validation is a meticulous and rigorous phase of psychometric properties evaluation. The most important of these properties are validity and reliability, which means, respectively, the "conclusions that can be drawn from the results of a test or scale" and the "overall reproducibility of measures obtained from an assessment tool or scale that can be evaluated" ${ }^{27}$ The confirmation of those psychometric properties are necessary once assessment tools are only valid for a specific purpose, in a determined group of respondents and in a given context. ${ }^{24}$ Because of that, most of the ICU pain scales were tested in different populations and languages, in order to prove their validity and applicability in general situations..$^{24}$ Description of each scale, population and languages used for validation are shown in Table 1.

\section{Critical-care pain observational tool (CPOT)}

CPOT is the most used behavior scale for pain assessment in patients unable to self-report. It was first validated in 2006 by Gélinas et a ${ }^{29}$ in cardiac surgery patients from Canada. ${ }^{29}$ This tool includes four behaviors, ie, facial expressions, movements, muscle tension, and ventilator compliance, and can be applied in intubated or non-intubated subjects. ${ }^{29}$ CPOT was tested and validated in many populations $\mathrm{s}^{30-46}$ and languages ${ }^{47-61}$ (Table 1), it has many psychometric properties already tested.

Interrater reliability (French version: weighted Kappa 0.52-0.8829; English version: intraclass coefficient [ICC] $0.80-0.93^{50}$; Spanish version: kappa 0.79 and $1.00,{ }^{61}$ Swedish version: ICC $0.84,{ }^{57}$ internal consistency (Cronbach $\alpha$ coefficients from $0.31-0.81^{57}$, sensitivity $(67-86 \%$ in two different ICU samples, ${ }^{33,50}$ ) specificity (78-83\% in two different ICU samples, ${ }^{33,50}$ ) feasibility (ICU nurses agreed that it was feasible and quick to use, simple to understand and easy to complete) ${ }^{62}$ criterion and discriminant validity were supported by different studies that confirm the effectiveness of this scale for ICU pain assessment. ${ }^{27}$

A previous study that performed CPOT implementation as a pain measurement routine in an ICU evidenced a positive influence on pain management (use of analgesics and sedatives) after pain assessment phase. ${ }^{63}$ In this study, French version of CPOT was applied by nurses for 12 months in ICU population, composed by medical and trauma patients $(\mathrm{n}=30) \cdot{ }^{63}$ Medical files were retrospectively analyzed to collect pain measurement and pain management data at three different times: pre-implementation, three months post-implementation and 12 months postimplementation. An increase on reports of pain assessment and on behaviors indicative of the presence of pain was evidenced. Alternatively, there was a decrease in the number of analgesics, propofol and morphine bolus administered after CPOT implementation. ${ }^{63}$ 
Table I Characteristics of pain assessment tools used in the intensive care unit

\begin{tabular}{|c|c|c|c|}
\hline Instrument & Score & Population & Language \\
\hline Critical-care pain & Each domain: 0-2 & Cardiac surgery ${ }^{29,30,33,34,38,40,41,43}$ & French $^{29}$ \\
\hline observational tool & Total score: 0 (no pain) to 8 (most pain) ${ }^{29}$ & Non-agitated patients ${ }^{31}$ & English ${ }^{50}$ \\
\hline \multirow[t]{10}{*}{$(\mathrm{CPOT})^{29}$} & & Neurosurgery ${ }^{32,45}$ & Turkish $^{47}$ \\
\hline & & Brain injury ${ }^{35,36,39}$ & Chinese ${ }^{48,51,55,56}$ \\
\hline & & Delirium $^{37,44}$ & Polish ${ }^{52,53}$ \\
\hline & & Surgical wards ${ }^{42}$ & Korean $^{54}$ \\
\hline & & Burned $^{46}$ & Swedish $^{57}$ \\
\hline & & & Finnish ${ }^{58}$ \\
\hline & & & Dutch $^{59}$ \\
\hline & & & Italian 60 \\
\hline & & & Danish $^{49}$ \\
\hline & & & Spanish \\
\hline Behavioral pain scale & Each domain: $1-4$ & Non-intubated ${ }^{75}$ & French $^{64}$ \\
\hline \multirow[t]{8}{*}{$(B P S)^{64}$} & Total score: 3 (no pain) to 12 (most pain) ${ }^{64}$ & Traumatic brain injury $y^{35,76-78}$ & English ${ }^{65,74}$ \\
\hline & & & Chinese ${ }^{68,69}$ \\
\hline & & & Swedish ${ }^{70}$ \\
\hline & & & Polish $^{53}$ \\
\hline & & & Finnish $^{58}$ \\
\hline & & & Dutch $^{73}$ \\
\hline & & & Portuguese ${ }^{66,67,71}$ \\
\hline & & & Spanish $^{72}$ \\
\hline Behavioral pain assessment tool & Eight dichotomized behavior items: present or absent ${ }^{83}$ & ICU patients of 28 countries $^{83}$ & 12 different \\
\hline$(\mathrm{BPAT})^{83}$ & & & languages ${ }^{83}$ \\
\hline Escala de conductas & Each domain: 0-2 & Traumatic brain injury ${ }^{85}$ & Spanish $28,84-86$ \\
\hline indicadoras de dolor & Total score: 0 (no pain); I-3: mild-moderate pain; 4-6: & Severe trauma ${ }^{84,86}$ & \\
\hline$(E S C I D)^{28}$ & moderate-severe pain; >6: very intense pain ${ }^{28}$ & & \\
\hline Nonverbal pain assessment tool & Each domain: $0-2$ & Cardiothoracic surgery, cardiology, & English $^{87}$ \\
\hline$(\mathrm{NPAT})^{87}$ & Total score: 0 (no pain) - 10 (most pain) ${ }^{87}$ & medical and surgical patients ${ }^{87}$ & \\
\hline Nonverbal pain scale & Each domain: $0-2$ & Trauma ${ }^{45,88,90-92}$ & English $^{88}$ \\
\hline \multirow[t]{4}{*}{$(N V P S)^{88}$} & Total score: 0 (no pain) to 10 (most pain) ${ }^{88}$ & Surgery ${ }^{80,88,90,92}$ & Iranian $^{92}$ \\
\hline & & Burned 88 & Finnish $^{58}$ \\
\hline & & Cardiac surgery ${ }^{41}$ & \\
\hline & & Neurological ${ }^{91,92}$ & \\
\hline Behavioral pain rating scale & Each domain: $0-3$ & Surgical ${ }^{93,94}$ & English $^{93}$ \\
\hline$(B P R S)^{93}$ & Total score: 0 (no pain) to 12 (most pain) ${ }^{93}$ & & \\
\hline Pain assessment and intervention & Total score: 0 (no pain) to 10 (most pain) ${ }^{95}$ & Surgical ${ }^{95}$ & English 95 \\
\hline notation & & & \\
\hline \multicolumn{4}{|l|}{ (PAIN) algorithm 95} \\
\hline Face, legs, activity, & Each item: 0-2 & Surgical ${ }^{97,98}$ & English ${ }^{97}$ \\
\hline cry, consolability & Total score: 0 (no pain) to 10 (most pain) ${ }^{97}$ & Neurological ${ }^{97,98}$ & \\
\hline$(\mathrm{FLACC})^{97}$ & & Cardiac surgery ${ }^{41}$ & \\
\hline Multidimensional objective pain & Behavioral dimension: 0 (none) to 3 (severe) & Medical patients ${ }^{101}$ & English ${ }^{101}$ \\
\hline assessment tool (MOPAT) ${ }^{100,101}$ & Physiologic dimension: 0 (no change) or I (change) ${ }^{100,101}$ & & \\
\hline
\end{tabular}

Despite its advantages, CPOT also has limitations. It is highlighted that traumatic brain injured patients present different facial expressions compared to other patients when exposed to nociceptive procedures; because of that, more studies are required to validate its use in this ICU population. ${ }^{27}$ In addition, the complex interpretation of the scores and the training to use this instrument is also reported as difficult. Despite these limitations, CPOT is considered a well-designed behavioral tool for use with nonverbal critically ill subjects.

\section{Behavioral pain scale (BPS)}

BPS is the second most used pain scale in ICUs. It evaluates three behavioral domains (facial expressions, movement of upper limbs, and compliance with ventilation $)^{64}$ and it is valid in different languages ${ }^{53,58,64-74}$ and samples ${ }^{35,75-78}$ (Table 1). Psychometric properties, as internal consistency (Cronbach $\alpha$ coefficient ranged from 0.63 to $0.72,{ }^{65,74,79,80}$ ) interrater reliability (Kappa coefficient ranged from $0.67-0.83^{64,79,81}$ and ICC of $0.95,{ }^{65}$ ) sensitivity, specificity, criterion and 
discriminant validity (significant increases of 2-3 points in BPS scores following nociceptive procedures ${ }^{64,65,69,74,79,80}$ ) were supported previously and confirm the advantages of BPS use, including frequency of pain assessments and patient's positive outcomes. ${ }^{64,65,74,82}$

BPS implementation in ICUs resulted in improvements for pain management and patients outcomes. Some items can be interpreted in a different way by users, which consists as a limitation in the practice. ${ }^{27}$ As an example of confounders, the items "movement of upper limbs" can be confused with muscle tension, and "compliance with ventilator" is defined by professionals as the less clear item described in this scale. ${ }^{26}$

\section{Behavioral pain assessment tool (BPAT)}

BPAT is a new instrument created in 2017 by Gélinas et al and validated in 28 countries. ${ }^{83}$ While other scales as CPOT requires interpretation of scores that is more complex than identifying when behaviors are present or absent, BPAT was created for use in a multinational study of procedural pain in ICU patients ${ }^{83}$ It is an eight-item scale composed of names and descriptions of facial expressions (accompanied by a picture), verbal responses (moaning and verbal complaints of pain) and body muscle responses (rigidity and clenched fists). These behavior items are dichotomized, that is, "present" or "absent" are possible answers to provide an easy and viable use for clinicians. ${ }^{83}$

In this validation study, interrater reliability (Kappa coefficients from 0.43-0.60), discriminant, criterion and convergent validity, sensitivity and specificity (61.8-75.1\%) were assessed..$^{83}$ Unlike other scales such as BPS and CPOT, BPAT does not have an item related to compliance with mechanical ventilation, which can be considered an advantage, once this is a confounder item. Authors who developed this instrument describe the necessity of further tests for different ICU patients groups for further validation in daily ICU practice. ${ }^{83}$

\section{Escala de conductas indicadoras de dolor (ESCID) Behavioural Indicators of Pain Scale}

ESCID is a Spanish instrument created and validated to assess pain in critically ill, nonverbal and mechanically ventilated patients. ${ }^{28}$ Four items are considered in this scale: facial muscles ("musculatura facial"/"facial expression"), tranquility ("tranquilidad"/"calmness"), muscle tone ("tono muscular"/"muscle tone"), adaptation to mechanical ventilation ("adaptación a ventilación mecánica"/"compliance with mechanical ventilation") and comfort ("confortabilidad"/ "Consolability"), scored from 0-2. ${ }^{28}$
Reliability (Cronbach $\alpha$ ranged from 0.70 to 0.80 ) and validity (good correlation between BPS and ESCID scores Pearson correlation from 0.94-0.97) were tested first in a general $\mathrm{ICU}^{28}$ and later in different sample, as severe trauma and brain injury patients. ${ }^{84-86}$ ESCID is a valid and applicable instrument, ${ }^{28}$ it is available only in Spanish, which is a limitation for clinicians in other countries with different languages. In addition to that limitation, authors recommend that ESCID should be tested in other situations such as painful and nonpainful procedures. ${ }^{28}$

\section{Nonverbal pain assessment tool (NPAT)}

NPAT was developed by Klein et al in 2010, based on behaviors commonly observed in ICU daily practice. ${ }^{87}$ Five categories are assessed by this tool: emotion (affective response to a situation), movement (change in the placement and positioning of the body and extremities when not engaged in any care activities), verbal cues (sound cues or vocalizations from the patient other than speech), facial cues (facial expressions) and positioning/guarding (body responses that imply a protection of the body from contact with external touch).$^{87}$

Some limitations are reported about this three-phase study conducted to validate this scale: (1) no information was provided about the occasions when pain was assessed; (2) the third study phase was conducted with a different sample; and (3) low correlation coefficients were found between NPAT scores and self-reports of pain intensity. ${ }^{27}$ These limitations suggest that more studies are necessary to prove NPAT validity and its applicability in ICUs.

\section{Nonverbal pain scale (NVPS)}

NVPS is a multidimensional instrument, initially modified from face, legs, activity, cry, consolability (FLACC) scale (NVPS-I) by Odhner et al in $2003^{88}$ and later revised (NVPS-R). ${ }^{27}$ It is composed of three behavioral (face, activity/movement, and guarding) and two physiological indicators (Physiologic I - blood pressure, heart rate, respiratory rate and Physiologic II - skin temperature, pupil dilation, perspiration, flushing, pallor). ${ }^{88}$

Interrater agreement and discriminant validity of NVPS were supported in previous studies..$^{80,89,90} \mathrm{It}$ is also known that after NVPS implementation in a critical care setting, patients' ratings of their pain experiences, documentation by nurses, and nurses' increased confidence in assessing pain in nonverbal patients were improved. ${ }^{91}$ Good applicability and validity (Cronbach $\alpha$ coefficients ranged from $0.36-0.75$ and ICC ranged from 0.60-0.76) in different samples $s^{41,45,48,80,88,90,91}$ and languages ${ }^{58,88,92}$ encourages use of NVPS in clinical practice. 


\section{Pain assessment and intervention notation (PAIN)} algorithm

PAIN algorithm is a multidimensional instrument developed to be tested by critical care nurses..$^{95}$ It is divided into three parts: (1) pain assessment (presence or absence of six behavioral domains - facial expression, movement, posture, vocal sounds, pallor and perspiration - and three physiologic indicators - heart rate, blood pressure, respiration); (2) assessment of patient's ability to tolerate opioids; and (3) guidelines for analgesic treatment decisions and documentation. ${ }^{26,95}$

As a multidimensional instrument, PAIN algorithm appears to be more than a single pain scale; it can be considered as a pain management educational tool. ${ }^{27}$ Despite psychometric properties as content and convergent validity were tested in this instrument, other properties were not previously reported, including internal consistency, interrater reliability and construct validity. ${ }^{26,27}$ Besides this weakness, the length of PAIN algorithm and the use of non-standardized assessment method based only on nurses' judgement and experience limits the use of this tool in clinical practice..$^{26,27}$

\section{Face, legs, activity, cry, consolability (FLACC)}

FLACC scale is a unidimensional instrument largely used in pediatric population with cognitive impairment, developed in 1997 by Merkel et al ${ }^{96}$ In critically ill adults, FLACC validity was tested by Voepel-Lewis et al in a recent study, ${ }^{97}$ which showed a good interrater reliability (ICC ranged from 0.72 to 0.98 ), internal consistency (Cronbach $\alpha=0.882$ ), criterion and construct validity. ${ }^{97}$ Previously, FLACC was used in comparison to other pain assessment instruments and was tested in different samples. ${ }^{41,98}$

Each item named in this scale (Face, Legs, Activity, Cry, Consolability) is rated from 0 to 2 , with a maximum possible score of 10 points. ${ }^{96}$ In the ICU adult sample, the "cry" category presented poor correlation with other categories and consequently lowered the internal consistency of this tool, ${ }^{97}$ suggesting that this item may not be relevant for adult patients. ${ }^{27}$ From that, it is suggested that the "cry item" should be modified in this scale, as occurred in FLACC in pediatrics. ${ }^{99}$ Despite the good results on psychometric analysis, further studies are necessary to establish FLACC reliability and validity for use in adult ICU patients. ${ }^{27}$

\section{Multidimensional objective pain assessment tool (MOPAT)}

MOPAT is a multidimensional scale developed and firstly validated to nonresponsive hospice patients in 2011 by McGuire et al. ${ }^{100}$ This instrument is composed of two subscales or dimensions (behavioral and physiological). The behavioral dimension has four indicators of acute pain (restless, tense muscles, frowning/grimacing, patient sounds), which are rated from 0 (none) to 3 (severe). The physiologic dimension has also four indicators (blood pressure, heart rate, respirations and diaphoresis), which are rated as 0 (no change) or 1 (change), according to patients' usual values of these physiologic indicators. ${ }^{100,101}$

Recently, MOPAT psychometric properties were tested in critically ill patients from a medical ICU. ${ }^{101}$ For reliability, internal consistency was considered moderate in this sample and interrater reliability agreement was reasonable for both dimensions and for total score. ${ }^{101}$ Validity results suggest that MOPAT is sensitive to measure acute pain in noncommunicative patients and that it is a quick and easy to use instrument. ${ }^{101}$

These results are based on a single study performed with a small sample. Due to that, studies with a larger ICU sample could improve psychometric properties' values and consolidate MOPAT use in practice.

\section{Vital signs}

The use of vital signs, blood pressure (BP), heart rate (HR) and respiratory rate (RR), by nurses in ICUs is a common practice due to the easy access through continuous monitoring. ${ }^{102}$ In a previous validity test of this pain assessment method for mechanically ventilated patients, a discriminant validity was supported, once there was higher HR and BP during a painful procedure (suctioning) than before and after this same or a nonnociceptive procedure. ${ }^{103}$ However, this same study concluded that there was no significant correlation between patients' self-report of pain intensity and HR and BP values, ${ }^{103}$ which instigates the research about the real validity of this method.

Vital signs validity as a pain assessment method is not supported by previous studies, ${ }^{3,102}$ since it is considered an unstable measure. It means that vital signs can increase, decrease or remain stable in ICU patients depending on their clinical condition. ${ }^{3,102}$ In addition, it is also proved that changes in physiological parameters can be caused by fear, anxiety and other psychological stressors ${ }^{102}$ or can be suppressed by analgesia. ${ }^{104}$ Because of these multifactorial causes of change, this is not a reliable pain measure.

This validity weakness was evidenced in many ICU samples, such as cardiac, ${ }^{105}$ brain $^{106}$ and general postsurgical ${ }^{107}$ patients. In all these studies, vital signs were not recommended as a valid measure, once they do not predict the presence of pain. Considering this poor evidence about vital signs utility for pain assessment, it should be used with caution, ${ }^{102}$ associated with objective measures (behavioral 
scales) for complete and reliable information about patient's pain intensity.

\section{Bispectral index (BIS)}

Brain activity in different cortical areas during pain and nociception integration can be measured by a specific technique named bispectral index (BIS). ${ }^{108}$ It consists of a noninvasive technology that provides, by the use of electrodes placed on the frontal and temporal areas, a cortical activity value that ranges from 0 (no cortical activity) to 100 (completely awake). ${ }^{109}$ This value (BIS) is calculated from a proprietary algorithm that interprets cortical activity (electroencephalography, EEG) and corrugator supercilii muscle activity (electromyography, EMG). ${ }^{109}$

The use of BIS for pain assessment has been studied in ICUs and surgery rooms. ${ }^{109}$ In mechanically ventilated patients, an increase in BIS value was evidenced during routine nociceptive procedures, ${ }^{110-113}$ such as endotracheal suctioning and mobilization, that can be reversed by opioids, neuromuscular blocking agents and sedative effects. ${ }^{10-112}$

This method appears promising due to its precision and technological value. However, BIS index was not primarily developed to measure pain, ${ }^{24}$ which implies in a lack of validated application method and standard values for ICU population. From that, it is suggested that further research with larger samples is needed to confirm BIS index clinical utility in ICUs. ${ }^{24}$

\section{Pupillary reflex/pupillometry}

The pupillary dilatation reflex (PDR) is known as a sympathetic reflex that dilates the pupil in response to noxious stimuli. ${ }^{114}$ Due to the autonomic nervous system influence on pain, pupillary responses have been studied as a method to detect its sign. ${ }^{115}$ It has been proposed as an alternative instrument for pain assessment during anesthesia for surgery or out of the surgery room. ${ }^{112,116-118}$

High sensitivity, specificity and positive correlation between pupillometry and pain scores were demonstrated in postoperative patients. ${ }^{116}$ Other studies show that PDR can predict quality of analgesia before procedural pain in ICU. ${ }^{117}$ However, PDR is not specific for pain and some conditions, as fear and anxiety, can confound results and should be considered during assessment. ${ }^{102}$ Future research is encouraged to describe specific conditions to use PDR with nonverbal ICU patients. ${ }^{102}$

\section{Skin conductance}

Palmar sweat gland activation is known as a physiological response to an increase on sympathetic nervous system activity due to emotional stress. ${ }^{119}$ This response also occurs when nociceptive stimulation is performed in the organism, resulting in an increased number of skin conductance fluctuations (NSCF). ${ }^{119,120}$

In order to measure this ongoing palmar sweat gland activation in response to pain (or emotional stress), a monitor and electrodes placed on the palmar surface of patient's hand are used. ${ }^{121}$ In ICUs, this procedure is reported in a previous study ${ }^{121}$ as a pain assessment method. It was evidenced that in critically ill intubated patients NSCF values increased during painful stimulation. ${ }^{121}$ However, NSCF data were not associated with pain behaviors and facial expressions in these patients, ${ }^{121}$ which is a weakness on validity for this method.

A recent study performed in a postsurgical ICU evidenced that skin conductance analgesimeter index (SCAI) and hemodynamic variables (BP and HR) significantly increased during painful procedures. ${ }^{122} \mathrm{~A}$ negative and significant correlation between SCAI and sedation level (Ramsay scale scores), was also observed which means that the higher the sedation level, the lower is the ability to express painful behaviors. ${ }^{122}$ Based on these results, authors defined that skin conductance analgesimeter has good properties for detecting pain in critically ill subjects. ${ }^{122}$

The fact that sympathetic nervous system can be altered by many factors limits the reliability of skin conductance as an isolated pain assessment method. While it is still a new assessment instrument, more evidence is needed to be well established in the clinical practice.

\section{Discussion}

This review emphasizes the use of validated and standardized instruments for pain assessment in mechanically ventilated patients. It is shown that behavioral scales are the most commonly used methods, followed by observation of vital signs. This last one is known as an unreliable procedure, as pain is not the only phenomena that can alter physiologic parameters.

In a recent perspective, new technological trends have been used for pain intensity measurement in ICUs that include pupillometry, BIS index and skin conductance analysis. Besides these advances, these instruments demand new research to confirm their validity and applicability in practice.

Previous reviews ${ }^{24,102,123}$ also describe these assessment methods as the best practice for pain evaluation in ICUs. Objective pain behavior scales, vital signs and BIS analysis were first cited in the last two decades ${ }^{123}$ as good techniques for pain and sedation assessment, with further studies necessary to prove their efficacy. In recent reviews, ${ }^{24,102}$ these 
instruments are well described, with details about psychometric properties, applicability, strengths and weaknesses of each item. This approach stimulates clinical use and knowledge application in the clinical practice, which is the aim of this review.

Specifically, reviews were previously published as a guide for clinicians and researchers with the main topics about behavioral scales. ${ }^{26,27,124-126}$ These studies reinforce the importance of their pain assessment tools and their use by nurses and other health professionals in ICUs. In addition to those reviews, a guideline written by Barr et al $(2013)^{3}$ also demonstrates pain assessment and management strategies for critically ill patients, with emphasis on the clinical applicability and professionals' consciousness about pain measurement. Based on that, the present review also shows the importance of this review by the description of validated and standard tools.

An important point elucidated in this review is the low number of pain assessment methods for critically ill adults in comparison to other ICU population, such as neonatal and pediatric ventilated patients. Over 40 methods for assessing pain in infants are available, ${ }^{127}$ while only 13 tools are known for adult subjects. Many studies with a summary of pain in neonatal and pediatric ICUs are published, ${ }^{128-131}$ while published data for adults is scarce. ${ }^{24,102}$

This discrepancy can be justified by the high concern about pain in early life, once it is already evidenced that newborns and infants do not have descending inhibitory pathways developed, that is, endogenous pain control is not well stablished during childhood. ${ }^{132}$ Additionally, it is proved that infants are $30-50 \%$ more sensitive to pain than adults and have lower pain tolerance than older children. ${ }^{133}$ Another fact that justifies the major attention to pain in this population consists in the cognitive inability to express pain, that is accentuated by a clinical condition, as mechanical ventilation, that limits body expressions. These facts can explain the reduced number of pain assessment tool for adults, but do not justify this lack in the literature.

The scarcity of studies about this theme in adults is accompanied by the difficulty to establish a pain assessment routine in ICU. A recently published qualitative study ${ }^{134}$ analyzes the challenges faced by nurses when using pain assessment scales for patients unable to communicate. Difficulties reported in this study were "forgotten priority", "organizational barriers", "attitudinal barriers" and "barriers to knowledge", ${ }^{134}$ which represents internal (personal) and external (daily routine) reasons for a low quality pain evaluation.

Despite its widespread use, there are still some barriers for the use of behavioral scales in ICUs, such as the lack of knowledge, skepticism regarding the evidence or benefit, a nonintuitive result or the perception that positive points observed in previous studies are not generalizable to local practice. ${ }^{135}$ From this perspective, studies that summarize pain assessment techniques are useful to stimulate their use in the clinical setting. Because of that, reviews like this one are necessary to improve health professionals' knowledge and affinity with this theme.

Pain treatment is strongly associated to pain assessment. The measurement of pain signs (behaviors, hemodynamic parameters and others) is recommended to establish appropriate analgesia intervention. ${ }^{3}$ This is encouraged as a routine practice $^{3}$ to be performed by all ICU professionals, particularly those who directly assist patients (clinicians, nurses, physical therapists).

Gold standard pain assessment tools (behavioral scales) and new trends for pain measurement in mechanically ventilated patients (pupillometry, skin conductance and BIS index) were identified and described in this study. This review encourages research to develop studies about pain measurement in mechanically ventilated patients to improve scientific evidence about this theme. Moreover, the present review aims to stimulate evidence-based practice in ICUs, promoting pain assessment as the fifth vital sign and, as consequence, improving analgo-sedation routine.

\section{Conclusion}

This review showed that objective measures, such as behavioral scales, are the most commonly used in adults unable to self-report their pain. Vital signs recording is not recommended as a reliable technique, since not only pain can change these parameters. Pupillometry, skin conductance and BIS index are new trends which have been studied for use in ICUs, which need to be established as valid and reliable methods. Data from this review emphasizes the importance of pain measurement in mechanically ventilated patients and its consequences for an adequate pain management in ICUs.

\section{Disclosure}

The authors report no conflicts of interest in this work.

\section{References}

1. Marshall JC, Bosco L, Adhikari NK, et al. What is an intensive care unit? A report of the task force of the World Federation of Societies of Intensive and Critical Care Medicine. J Crit Care. 2017;37:270-276.

2. Ferri M, Zygun DA, Harrison A, Stelfox HT. Evidence-based design in an intensive care unit: End-user perceptions. BMC Anesthesiol. 2015;15(1):57.

3. Barr J, Fraser GL, Puntillo K, et al. Clinical practice guidelines for the management of pain, agitation, and delirium in adult patients in the intensive care unit. Crit Care Med. 2013;41(1):263-306. 
4. Merskey H, Bogduk N. Part III pain terms, a current list with definitions and notes on usage. Classification of chronic pain-descriptions of chronic pain syndromes and definitions of pain terms. Seattle: International Association for the Study of Pain: IASP Press; 1994:207-214.

5. Mehta S, Burry L, Martinez-Motta JC, et al. A randomized trial of daily awakening in critically ill patients managed with a sedation protocol: A pilot trial. Crit Care Med. 2008;36(7):2092-2099.

6. Burchardi H. Aims of sedation/analgesia. Minerva Anestesiol. 2004;70(4):137-143.

7. Egerod I. Cultural changes in ICU sedation management. Qual Health Res. 2009;19(5):687-696.

8. Egerod I, Christensen BV, Johansen L. Trends in sedation practices in Danish intensive care units in 2003: a national survey. Intensive Care Med. 2006;32(1):60-66.

9. Mattia C, Savoia G, Paoletti F, et al. SIAARTI recommendations for analgo-sedation in intensive care unit. Minerva Anestesiol. 2006;72(10):769.

10. Sessler CN, Varney K. Patient-focused sedation and analgesia in the ICU. Chest. 2008;133(2):552-565.

11. Strøm T, Martinussen T, Toft P. A protocol of no sedation for critically ill patients receiving mechanical ventilation: a randomised trial. Lancet. 2010;375(9713):475-480.

12. Egerod I, Jensen MB, Herling SF, Welling K-L. Effect of an analgosedation protocol for neurointensive patients: a two-phase interventional non-randomized pilot study. Crit Care. 2010;14(2):R71.

13. Arias-Rivera S, del Mar Sánchez-Sánchez M, Santos-Díaz R, et al. Effect of a nursing-implemented sedation protocol on weaning outcome. Crit Care Med. 2008;36(7):2054-2060.

14. Marshall J, Finn CA, Theodore AC. Impact of a clinical pharmacist-enforced intensive care unit sedation protocol on duration of mechanical ventilation and hospital stay. Crit Care Med. 2008;36(2): 427-433.

15. Quenot J-P, Ladoire S, Devoucoux F, et al. Effect of a nurse-implemented sedation protocol on the incidence of ventilator-associated pneumonia. Crit Care Med. 2007;35(9):2031-2036.

16. Puntillo KA, Max A, Timsit J-F, et al. Determinants of procedural pain intensity in the intensive care unit. The Europain ${ }^{\mathbb{R}}$ study. Am J Respir Crit Care Med. 2014;189(1):39-47.

17. Arroyo-Novoa CM, Figueroa-Ramos MI, Puntillo KA, et al. Pain related to tracheal suctioning in awake acutely and critically ill adults: a descriptive study. Intensive Crit Care Nurs. 2008;24(1):20-27.

18. Shannon K, Bucknall T. Pain assessment in critical care: what have we learnt from research. Intensive Crit Care Nurs. 2003;19(3):154-162.

19. Joffe AM, Hallman M, Gélinas C, Herr DL, Puntillo K. Evaluation and treatment of pain in critically ill adults. Semin Respir Crit Care Med. 2013;34(2):189-200.

20. Morone NE, Weiner DK. Pain as the fifth vital sign: exposing the vital need for pain education. Clin Ther. 2013;35(11):1728-1732.

21. Georgiou E, Hadjibalassi M, Lambrinou E, Andreou P, Papathanassoglou EDE. The impact of pain assessment on critically ill patients' outcomes: a systematic review. Biomed Res Int. 2015;2015(2):1-18.

22. Olsen BF, Rustøen T, Sandvik L, Jacobsen M, Valeberg BT. Results of implementing a pain management algorithm in intensive care unit patients: The impact on pain assessment, length of stay, and duration of ventilation. J Crit Care. 2016;36:207-211.

23. Payen J-F, Bosson J-L, Chanques G, Mantz J, Labarere J. Pain assessment is associated with decreased duration of mechanical ventilation in the intensive care unit: a post hoc analysis of the DOLOREA study. Anesthesiol. 2009;111(6):1308-1316.

24. Gélinas C. Pain assessment in the critically ill adult: Recent evidence and new trends. Intensive Crit Care Nurs. 2016;34:1-11.

25. Rahu MA, Grap MJ, Cohn JF, Munro CL, Lyon DE, Sessler CN. Facial expression as an indicator of pain in critically ill intubated adults during endotracheal suctioning. Am J Crit Care. 2013;22(5):412-422.

26. Li D, Puntillo K, Miaskowski C. A review of objective pain measures for use with critical care adult patients unable to self-report. J Pain. 2008;9(1):2-10.
27. Gelinas C, Puntillo KA, Joffe AM, Barr J. A validated approach to evaluating psychometric properties of pain assessment tools for use in nonverbal critically ill adults. Semin Respir Crit Care Med. 2013;34(2):153-168.

28. Latorre Marco I, Solís Muñoz M, Falero Ruiz T, et al. Validación de la Escala de Conductas Indicadoras de Dolor para valorar el dolor en pacientes críticos, no comunicativos y sometidos a ventilación mecánica: resultados del proyecto ESCID. [Validation of the Scale of Behavior Indicators of Pain (ESCID) in critically ill, non-communicative patients under mechanical ventilation: results of the ESCID scale] Enfermería Intensiva. 2011;22(1):3-12.

29. Gélinas C, Fillion L, Puntillo KA, Viens C, Fortier M. Validation of the critical-care pain observation tool in adult patients. Am J Crit Care. 2006;15(4):420-427

30. Boitor M, Fiola JL, Gélinas C. Validation of the critical-care pain observation tool and vital signs in relation to the sensory and affective components of pain during mediastinal tube removal in postoperative cardiac surgery intensive care unit adults. J Cardiovasc Nurs. 2016;31(5):425-432.

31. Chookalayia H, Heidarzadeh M, Hassanpour-Darghah M, Aghamohammadi-Kalkhoran M, Karimollahi M. The critical care pain observation tool is reliable in non-agitated but not in agitated intubated patients. Intensive Crit Care Nurs. 2018;44:123-128.

32. Echegaray-Benites C, Kapoustina O, Gélinas C. Validation of the use of the critical-care pain observation tool (CPOT) with brain surgery patients in the neurosurgical intensive care unit. Intensive Crit Care Nurs. 2014;30(5):257-265.

33. Gélinas C, Harel F, Fillion L, Puntillo KA, Johnston CC. Sensitivity and specificity of the critical-care pain observation tool for the detection of pain in intubated adults after cardiac surgery. J Pain Symptom Manage. 2009;37(1):58-67.

34. Ghanbari A, Bahadorizadeh N, Farmanbar R, Kazemnejad E. Sensitivity and specificity of critical care pain observation tool used for intubated patients following open-heart surgery. Intensive Care Med. 2016;42(4):618-619.

35. Jendoubi A, Abbes A, Ghedira S, Houissa M. Pain measurement in mechanically ventilated patients with traumatic brain injury: Behavioral pain tools versus analgesia nociception index. Indian J Crit Care Med. 2017;21(9):585.

36. Joffe AM, Mcnulty B, Boitor M, Marsh R, Gélinas C. Validation of the critical-care pain observation tool in brain-injured critically ill adults. J Crit Care. 2016;36:76-80.

37. Kanji S, Macphee H, Singh A, et al. Validation of the critical care pain observation tool in critically ill patients with delirium. Crit Care Med. 2016;44(5):943-947.

38. Kiavar M, Azarfarin R, Totonchi Z, Tavakoli F, Alizadehasl A, Teymouri M. Comparison of two pain assessment tools, "facial expression" and "critical care pain observation tool" in intubated patients after cardiac surgery. Anesth Pain Med. 2016;6(1).

39. Lee K, Oh H, Suh Y, Seo W. Patterns and clinical correlates of pain among brain injury patients in critical care assessed with the critical care pain observation tool. Pain Manag Nurs. 2013;14(4): 259-267.

40. Linde SM, Badger JM, Machan JT, et al. Reevaluation of the criticalcare pain observation tool in intubated adults after cardiac surgery. Am J Crit Care. 2013;22(6):491-497.

41. Marmo L, Fowler S. Pain assessment tool in the critically ill post-open heart surgery patient population. Pain Manag Nurs. 2010;11(3):134-140.

42. Rafiei M, Ghadami A, Irajpour A, Feizi A. Validation of critical care pain observation tool in patients hospitalized in surgical wards. Iran J Nurs Midwifery Res. 2016;21(5):464.

43. Rijkenberg S, Stilma W, Bosman RJ, van der Meer NJ, van der Voort PHJ. Pain measurement in mechanically ventilated patients after cardiac surgery: comparison of the behavioral pain scale (BPS) and the critical-care pain observation tool (CPOT). J Cardiothorac Vasc Anesth. 2017;31(4):1227-1234. 
44. Rijkenberg S, van der Voort PHJ. Can the critical-care pain observation tool (CPOT) be used to assess pain in delirious ICU patients? $J$ Thorac Dis. 2016;8(5):E285-E287.

45. Topolovec-Vranic J, Gélinas C, Li Y, et al. Validation and evaluation of two observational pain assessment tools in a trauma and neurosurgical intensive care unit. Pain Res Manag. 2013;18(6):e107-e114.

46. Wibbenmeyer L, Sevier A, Liao J, et al. Evaluation of the usefulness of two established pain assessment tools in a burn population. J Burn Care Res. 2011;32(1):52-60.

47. Aktaş YY, Karabulut N. A Turkish version of the critical-care pain observation tool: reliability and validity assessment. J Perianesth Nurs. 2017;32(4):341-351.

48. Cheng LH, Tsai YF, Wang CH, Tsay PK. Validation of two Chineseversion pain observation tools in conscious and unconscious critically ill patients. Intensive Crit Care Nurs. 2018;44:115-122.

49. Frandsen JB, O'Reilly Poulsen KS, Laerkner E, Stroem T. Validation of the Danish version of the critical care pain observation tool. Acta Anaesthesiol Scand. 2016;60(9):1314-1322.

50. Gélinas C, Johnston C. Pain assessment in the critically ill ventilated adult: validation of the critical-care pain observation tool and physiologic indicators. Clin J Pain. 2007;23(6):497-505.

51. Hsiung N-H, Yang Y, Lee MS, et al. Translation, adaptation, and validation of the behavioral pain scale and the critical-care pain observational tools in Taiwan. J Pain Res. 2016;9:661.

52. Kotfis K, Zegan-Barańska M, Strzelbicka M, et al. Validation of the Polish version of the critical care pain observation tool (CPOT) to assess pain intensity in adult, intubated intensive care unit patients: the POL-CPOT study. Arch Med Sci. 2018;14(4):880-889.

53. Kotfis K, Zegan-Barańska M, Szydłowski Ł, Żukowski M, Ely EW. Methods of pain assessment in adult intensive care unit patientsPolish version of the CPOT (critical care pain observation tool) and BPS (behavioral pain scale). Anaesthesiol Intensive Ther. 2017;49(1): 66-72.

54. Kwak E-M, Oh H. Validation of a Korean translated version of the critical care pain observation tool (CPOT) for ICU patients. J Korean Acad Nurs. 2012;42(1):76.

55. Li Q, Wan X, Gu C, et al. Pain assessment using the critical-care pain observation tool in Chinese critically ill ventilated adults. J Pain Symptom Manage. 2014;48(5):975-982.

56. Liu Y, Li L, Herr K. Evaluation of two observational pain assessment tools in Chinese critically ill patients. Pain Medicine. 2015;16(8):1622-1628.

57. Nürnberg Damström D, Saboonchi F, Sackey PV, Björling G. A preliminary validation of the Swedish version of the critical-care pain observation tool in adults. Acta Anaesthesiol Scand. 2011;55(4): 379-386.

58. Pudas-Tähkä S-M, Axelin A, Aantaa R, Lund V, Salanterä S. Translation and cultural adaptation of an objective pain assessment tool for Finnish ICU patients. Scand J Caring Sci. 2014;28(4):885-894.

59. Stilma W, Rijkenberg S, Feijen HM, Maaskant JM, Endeman H. Validation of the Dutch version of the critical-care pain observation tool. Nurs Crit Care. 2015 Dec 22. doi: 10.1111/nicc.12225. [Epub ahead of print]

60. Sulla F, de Souza Ramos N, Terzi N, et al. Validation of the Italian version of the critical pain observation tool in brain-injured critically ill adults. Acta Biomed. 2017;88(5-S):48-54.

61. Vázquez M, Pardavila M-I, Lucia M, Aguado Y, Margall M, Asiain MC. Pain assessment in turning procedures for patients with invasive mechanical ventilation. Nurs Crit Care. 2011;16(4):178-185.

62. Gélinas C. Nurses' evaluations of the feasibility and the clinical utility of the critical-care pain observation tool. Pain Manag Nurs. 2010;11(2):115-125.

63. Gélinas C, Arbour C, Michaud C, Vaillant F, Desjardins S. Implementation of the critical-care pain observation tool on pain assessment/ management nursing practices in an intensive care unit with nonverbal critically ill adults: a before and after study. Int J Nurs Stud. 2011;48(12):1495-1504.
64. Payen J-F, Bru O, Bosson J-L, et al. Assessing pain in critically ill sedated patients by using a behavioral pain scale. Crit Care Med. 2001;29(12): 2258-2263.

65. Aïssaoui Y, Zeggwagh AA, Zekraoui A, Abidi K, Abouqal R. Validation of a behavioral pain scale in critically ill, sedated, and mechanically ventilated patients. Anesth Analg. 2005;101(5):1470-1476.

66. Azevedo-Santos IF, Alves IGN, Badauê-Passos D, Santana-Filho VJ, Desantana JM. Psychometric analysis of behavioral pain scale Brazilian version in sedated and mechanically ventilated adult patients: a preliminary study. Pain Practice. 2016;16(4):451-458.

67. Azevedo-Santos IF, Alves IGN, Cerqueira Neto ML, Badauê-Passos D, Santana-Filho VJ, Santana JM. Validation of the Brazilian version of behavioral pain scale in adult sedated and mechanically ventilated patients. Rev Bras Anestesiol. 2017;67(3):271-277.

68. Chen J, Lu Q, Wu X-Y, An Y-Z, Zhan Y-C, Zhang H-Y. Reliability and validity of the Chinese version of the behavioral pain scale in intubated and non-intubated critically ill patients: Two cross-sectional studies. Int J Nurs Stud. 2016;61:63-71.

69. Chen Y-Y, Lai Y-H, Shun S-C, Chi N-H, Tsai P-S, Liao Y-M. The Chinese behavior pain scale for critically ill patients: Translation and psychometric testing. Int J Nurs Stud. 2011;48(4):438-448.

70. Hylén M, Akerman E, Alm-roijer C, Idvall E. Behavioral pain scale - translation, reliability, and validity in a Swedish context. Acta Anaesthesiologica Scandinavica. 2016;60(6):821-828.

71. Morete MC, Mofatto SC, Pereira CA, Silva AP, Odierna MT. Translation and cultural adaptation of the Brazilian Portuguese version of the behavioral pain scale. Rev Bras Ter Intensiva. 2014;26(4): 373-378.

72. Navarro-Colom M, Sendra-Lluis MA, Castillo-Masa AM, Robleda G. Fiabilidad interobservador y consistencia interna de la Behavioral Pain Scale en pacientes con ventilación mecánica [Intraobserver reliability and internal consistency of the Behavioral Pain Scale in mechanicallyventilated patients]. Enfermería Intensiva. 2015;26(1):24-31.

73. van der Woude MC, Bormans L, Hofhuis JG, Spronk PE. Current use of pain scores in dutch intensive care units: a postal survey in the Netherlands. Anesth Analg. 2016;122(2):456-461.

74. Young J, Siffleet J, Nikoletti S, Shaw T. Use of a behavioural pain scale to assess pain in ventilated, unconscious and/or sedated patients. Intensive Crit Care Nurs. 2006;22(1):32-39.

75. Chanques G, Payen J-F, Mercier G, et al. Assessing pain in nonintubated critically ill patients unable to self report: an adaptation of the behavioral pain scale. Intensive Care Med. 2009;35(12): 2060-2067.

76. Dehghani $\mathrm{H}$, Tavangar $\mathrm{H}$, Ghandehari A. Validity and reliability of behavioral pain scale in patients with low level of consciousness due to head trauma hospitalized in intensive care unit. Arch Trauma Res. 2014;3(1).

77. Ribeiro CJN, Bezerra DS, Lima AGCF, et al. Pain during tracheal aspiration in patients with traumatic brain injury undergoing mechanical ventilation. Revista Dor. 2017;18(4):332-337.

78. Yu A, Teitelbaum J, Scott J, et al. Evaluating pain, sedation, and delirium in the neurologically critically ill-feasibility and reliability of standardized tools. Crit Care Med. 2013;41(8):2002-2007.

79. Ahlers SJ, van der Veen AM, van Dijk M, Tibboel D, Knibbe CA. The use of the behavioral pain scale to assess pain in conscious sedated patients. Anesth Analg. 2010;110(1):127-133.

80. Juarez P, Bach A, Baker M, et al. Comparison of two pain scales for the assessment of pain in the ventilated adult patient. Dimens Crit Care Nurs. 2010;29(6):307-315.

81. Ahlers SJGM, van Gulik L, van der Veen AM, et al. Comparison of different pain scoring systems in critically ill patients in a general ICU. Critical Care. 2008;12(1):R15.

82. Chanques G, Jaber S, Barbotte E, et al. Impact of systematic evaluation of pain and agitation in an intensive care unit. Crit Care Med. 2006;34(6):1691-1699.

83. Gélinas C, Puntillo KA, Levin P, Azoulay E. The behavior pain assessment tool for critically ill adults: a validation study in 28 countries. Pain. 2017;158(5):811-821. 
84. López CL, Pérez MM, Vela ST, et al. Aplicación de la Escala de conductas indicadoras de dolor (ESCID) en el paciente con trauma grave no comunicativo y ventilación mecánica [Pain behaviour indicator scale (ESCID) application in severe non-communicative trauma patients receiving mechanical ventilation]. Enfermeria Intensiva. 2013;24(4):137-144.

85. López-López C, Murillo-Pérez MA, Morales-Sánchez C, et al. Valoración del dolor en la aspiración de secreciones traqueales en pacientes con traumatismo craneal mediante la Escala de conductas indicadoras de dolor (ESCID) [Pain assessment of tracheal suctioning on brain injury patients by pain behavioral indicator scale (ESCID)]. Enfermería Intensiva. 2014;25(3):114-121.

86. López-López C, Pérez-Pérez T, Beneit-Montesinos JV, et al. Pain assessment in mechanically ventilated, noncommunicative severe trauma patients. J Trauma Nurs. 2018;25(1):49-59.

87. Klein DG, Dumpe M, Katz E, Bena J. Pain assessment in the intensive care unit: development and psychometric testing of the nonverbal pain assessment tool. Heart Lung. 2010;39(6):521-528.

88. Odhner M, Wegman D, Freeland N, Steinmetz A, Ingersoll GL. Assessing pain control in nonverbal critically ill adults. Dimens Crit Care Nurs. 2003;22(6):260-267.

89. Chanques G, Pohlman A, Kress JP, et al. Psychometric comparison of three behavioural scales for the assessment of pain in critically ill patients unable to self-report. Crit Care. 2014;18(5):R160.

90. Kabes AM, Graves JK, Norris J. Further validation of the nonverbal pain scale in intensive care patients. Crit Care Nurse. 2009;29(1): 59-66.

91. Topolovec-Vranic J, Canzian S, Innis J, Pollmann-Mudryj MA, Mcfarlan AW, Baker AJ. Patient satisfaction and documentation of pain assessments and management after implementing the adult nonverbal pain scale. Am J Crit Care. 2010;19(4):345-354.

92. Chookalayi H, Heidarzadeh M, Hasanpour M, Jabrailzadeh S, Sadeghpour F. A study on the psychometric properties of revised-nonverbal pain scale and original-nonverbal pain scale in Iranian nonverbalventilated patients. Indian J Crit Care Med. 2017;21(7):429-435.

93. Mateo O, Krenzischek D. A pilot study to assess the relationship between behavioral manifestations and self-report of pain in postanesthesia care unit patients. $J$ Post Anesth Nurs. 1992;7(1):15-21.

94. Webb M, Kennedy M. Behavioral responses and self-reported pain in postoperative patients. J Post Anesth Nurs. 1994;9(2):91-95.

95. Puntillo KA, Miaskowski C, Kehrle K, Stannard D, Gleeson S, Nye P. Relationship between behavioral and physiological indicators of pain, critical care patients' self-reports of pain, and opioid administration. Crit Care Med. 1997;25(7):1159-1166.

96. Merkel SI, Voepel-Lewis T, Shayevitz JR, Malviya S. The FLACC: a behavioral scale for scoring postoperative pain in young children. Pediatr Nurs. 1997;23(3):293-297.

97. Voepel-Lewis T, Zanotti J, Dammeyer JA, Merkel S. Reliability and validity of the face, legs, activity, cry, consolability behaviora tool in assessing acute pain in critically ill patients. Am J Crit Care. 2010;19(1):55-61.

98. Buttes P, Keal G, Cronin SN, Stocks L, Stout C. Validation of the critical-care pain observation tool in adult critically ill patients. Dimens Crit Care Nurs. 2014;33(2):78-81.

99. Johansson M, Kokinsky E. The COMFORT behavioural scale and the modified FLACC scale in paediatric intensive care. Nurs Crit Care. 2009;14(3):122-130.

100. McGuire DB, Reifsnyder J, Soeken K, Kaiser KS, Yeager KA. Assessing pain in nonresponsive hospice patients: development and preliminary testing of the multidimensional objective pain assessment tool (MOPAT). J Palliat Med. 2011;14(3):287-292.

101. Wiegand DL, Wilson T, Pannullo D, et al. Measuring acute pain over time in the critically ill using the multidimensional objective pain assessment tool (MOPAT). Pain Manag Nurs. 2018;19(3):277-287.

102. Gélinas $\mathrm{C}$, Chanques G, Puntillo K. In pursuit of pain: recent advances and future directions in pain assessment in the ICU. Intensive Care Med. 2014;40(7):1009-1014.
103. Chen HJ, Chen YM. Pain assessment: validation of the physiologic indicators in the ventilated adult patient. Pain Manag Nurs. 2015;16(2):105-111.

104. Jeitziner MM, Schwendimann R, Hamers JP, Rohrer O, Hantikainen $\mathrm{V}$, Jakob SM. Assessment of pain in sedated and mechanically ventilated patients: an observational study. Acta Anaesthesiol Scand. 2012;56(5):645-654

105. Arbour C, Gélinas C. Are vital signs valid indicators for the assessment of pain in postoperative cardiac surgery ICU adults? Intensive Crit Care Nurs. 2010;26(2):83-90.

106. Kapoustina O, Echegaray-Benites C, Gélinas C. Fluctuations in vital signs and behavioural responses of brain surgery patients in the intensive care unit: are they valid indicators of pain? JAdv Nurs. 2014;70(11):2562-2576.

107. Asgar Pour H. Association between acute pain and hemodynamic parameters in a postoperative surgical intensive care unit. Aorn $J$. 2017;105(6):571-578.

108. Treede RD, Apkarian AV, Bromm B, Greenspan JD, Lenz FA. Cortical representation of pain: functional characterization of nociceptive areas near the lateral sulcus. Pain. 2000;87(2):113-119.

109. Coleman RM, Tousignant-Laflamme Y, Ouellet P, Parenteau-Goudreault É, Cogan J, Bourgault P. The use of the bispectral index in the detection of pain in mechanically ventilated adults in the intensive care unit: a review of the literature. Pain Res Manag. 2015;20(1):e33-e37.

110. Brocas E, Dupont H, Paugam-Burtz C, Servin F, Mantz J, Desmonts $\mathrm{JM}$. Bispectral index variations during tracheal suction in mechanically ventilated critically ill patients: effect of an alfentanil bolus. Intensive Care Med. 2002;28(2):211-213.

111. Gélinas C, Tousignant-LaflammeY, Tanguay A, Bourgault P. Exploring the validity of the bispectral index, the critical-care pain observation tool and vital signs for the detection of pain in sedated and mechanically ventilated critically ill adults: a pilot study. Intensive Crit Care Nurs. 2011;27(1):46-52.

112. Li D, Miaskowski C, Burkhardt D, Puntillo K. Evaluations of physiologic reactivity and reflexive behaviors during noxious procedures in sedated critically ill patients. J Crit Care. 2009;24(3):472.e9-47472.

113. Robleda G, Roche-Campo F, Membrilla-Martínez L, et al. Evaluation of pain during mobilization and endotracheal aspiration in critical patients. Med Intensiva. 2016;40(2):96-104.

114. Yang LL, Niemann CU, Larson MD. Mechanism of pupillary reflex dilation in awake volunteers and in organ donors. Anesthesiology. 2003;99(6):1281-1286

115. Larson MD, Kurz A, Sessler DI, Dechert M, Bjorksten AR, Tayefeh F. Alfentanil blocks reflex pupillary dilation in response to noxious stimulation but does not diminish the light reflex. Anesthesiology. 1997;87(4):849-855.

116. Aissou M, Snauwaert A, Dupuis C, Atchabahian A, Aubrun F, Beaussier M. Objective assessment of the immediate postoperative analgesia using pupillary reflex measurement: a prospective and observational study. Anesthesiology. 2012;116(5):1006-1012.

117. Paulus J, Roquilly A, Beloeil H, Théraud J, Asehnoune K, Lejus C. Pupillary reflex measurement predicts insufficient analgesia before endotracheal suctioning in critically ill patients. Crit Care. 2013;17(4):R161

118. Lukaszewicz AC, Dereu D, Gayat E, Payen D. The relevance of pupillometry for evaluation of analgesia before noxious procedures in the intensive care unit. Anesth Analg. 2015;120(6):1297-1300.

119. Storm $\mathrm{H}$. Changes in skin conductance as a tool to monitor nociceptive stimulation and pain. Curr Opin Anaesthesiol. 2008;21(6):796-804.

120. Storm H, Fremming A, Odegaard S, Martinsen OG, Morkrid L. The development of a software program for analyzing spontaneous and externally elicited skin conductance changes in infants and adults. Clin Neurophysiol. 2000;111(10):1889-1898.

121. Günther AC, Bottai M, Schandl AR, Storm H, Rossi P, Sackey PV. Palmar skin conductance variability and the relation to stimulation, pain and the motor activity assessment scale in intensive care unit patients. Crit Care. 2013;17(2):R51. 
122. Khanna P, Chandralekha C, Pandey RK, Sharma A. Pain assessment in the critically ill mechanically ventilated adult patients: comparison between skin conductance algesimeter index and physiologic indicators. Saudi J Anaesth. 2018;12(2):204.

123. Hamill-Ruth RJ, Marohn ML. Evaluation of pain in the critically ill patient. Crit Care Clin. 1999;15(1):35-54.

124. Cade $\mathrm{CH}$. Clinical tools for the assessment of pain in sedated critically ill adults. Nurs Crit Care. 2008;13(6):288-297.

125. Pudas-Tähkä SM, Axelin A, Aantaa R, Lund V, Salanterä S. Pain assessment tools for unconscious or sedated intensive care patients: a systematic review. J Adv Nurs. 2009;65(5):946-956.

126. Varndell W, Fry M, Elliott D. A systematic review of observational pain assessment instruments for use with nonverbal intubated critically ill adult patients in the emergency department: an assessment of their suitability and psychometric properties. J Clin Nurs. 2017;26(1-2):7-32.

127. Ranger M, Johnston CC, Anand KJ. Current controversies regarding pain assessment in neonates. Semin Perinatol. 2007;31(5):283-288.

128. Hall SJ. Paediatric pain assessment in intensive care units. Intensive Crit Care Nurs. 1995;11(1):20-25.
129. Harris J, Ramelet AS, van Dijk M, et al. Clinical recommendations for pain, sedation, withdrawal and delirium assessment in critically ill infants and children: an ESPNIC position statement for healthcare professionals. Intensive Care Med. 2016;42(6):972-986.

130. Oakes LL. Assessment and management of pain in the critically ill pediatric patient. Crit Care Nurs Clin North Am. 2001;13(2):281-295.

131. Maxwell LG, Malavolta CP, Fraga MV. Assessment of pain in the neonate. Clin Perinatol. 2013;40(3):457-469.

132. Fitzgerald M. What do we really know about newborn infant pain? Exp Physiol. 2015;100(12):1451-1457.

133. Young EE, D'agata A, Vittner D, Baumbauer KM. Neurobiological consequences of early painful experience: basic science findings and implications for evidence-based practice. J Perinat Neonatal Nurs. 2017;31(2):178-185.

134. Deldar K, Froutan R, Ebadi A. Challenges faced by nurses in using pain assessment scale in patients unable to communicate: a qualitative study. BMC Nurs. 2018;17(1):11.

135. Luk KH, Joffe AM. How painful could it be? The challenge of measuring pain in the intensive care unit. Anesth Analg. 2016;122(2):302-304.
Journal of Pain Research

\section{Publish your work in this journal}

The Journal of Pain Research is an international, peer reviewed, open access, online journal that welcomes laboratory and clinical findings in the fields of pain research and the prevention and management of pain. Original research, reviews, symposium reports, hypothesis formation and commentaries are all considered for publication.

\section{Dovepress}

The manuscript management system is completely online and includes a very quick and fair peer-review system, which is all easy to use. Visit http://www.dovepress.com/testimonials.php to read real quotes from published authors. 